\title{
Der IVDK - eine kurze Synopsis
}

\author{
The IVDK - A Short Synopsis
}

Autor

Institut

\section{A. Schnuch}

Informationsverbund Dermatologischer Kliniken (IVDK) an der Universitäts-Hautklinik Göttingen

\section{Bibliografie}

DOI $10.1055 / \mathrm{s}-0028-1119436$

Akt Dermatol 2009; 35:

94-98 ๑ Georg Thieme

Verlag KG Stuttgart · New York ISSN 0340-2541

Korrespondenzadresse

Prof. Dr. med. Axel Schnuch Zentrale des IVDK

Universitäts-Hautklinik

von-Siebold-Str. 3

37075 Göttingen

aschnuch@med.uni-

goettingen.de

\section{Zusammenfassung \\ $\nabla$}

Das multizentrische Projekt IVDK (Informationsverbund Dermatologischer Kliniken), an dem 50 Hautkliniken aus Deutschland, Österreich und der Schweiz beteiligt sind, hat die epidemiologische Erforschung der Kontaktallergien zum Ziel. Die bei der Diagnostik anfallenden Daten (u.a. anamnestische Angaben zum Beruf, Epikutantestergebnisse) werden seit 1989 zentral gespeichert. Entsprechend der Art der Datengewinnung - halbjährliche Datenlieferung der Partnerkliniken an die Zentrale, und kontinuierlich wachsender Datenbestand, der im August 2008 Daten von $>170000$ Patienten umfasste - lassen sich zwei Arten der Datennutzung unterscheiden: Nutzung als dynamisches Überwachungssystem, und Nutzung als ein statisches Register. Mit der Analyse von Registerdaten können Risikofaktoren (z.B.

Allergieabteilungen von 50 Hautkliniken Deutschlands, Österreichs und der Schweiz kooperieren in dem multizentrischen Projekt Informationsverbund Dermatologischer Kliniken (IVDK) bei der Erfassung, Dokumentation und zentralen Auswertung der Daten, die im Rahmen der Diagnostik der allergischen Kontaktdermatitis (ACD) erhoben werden. Die Zentrale des Projektes befindet sich an der Univ.-Hautklinik Göttingen. Das Ziel ist, eine solide Datenbasis für die primäre Prävention der ACD zu schaffen.

\section{Hintergrund und Rationale des Projektes \\ $\nabla$}

Etwa 7\% der Bevölkerung sind jährlich von der ACD betroffen (Bundesgesundheitssurvey 2000). Zwischen 15\% und 20\% der Allgemeinbevölkerung sind gegen mindestens eines der wichtigen Allergene sensibilisiert [1]. Unter gesundheitspo- bestimmte Berufe oder bestimmte Expositionen) identifiziert und quantifiziert werden, wie am Beispiel der Allergie gegen Aminoglykosid Antibiotika gezeigt wird. Es stellte sich heraus, dass Kanamycin mit einem tendenziell höheren Kontakt-Allergie-Risiko assoziiert ist als Gentamicin. Die halbjährlich neu eingehenden Daten (von etwa 6000 Patienten) erlauben demgegenüber Trendberechnungen mit dem vorrangigen Ziel, problematische Entwicklungen im Bereich der Sensibilisierungen zu identifizieren, wofür Beispiele (Allergie gegen Duftstoffe und andere) gegeben werden, um so die Grundlage für präventive Interventionen zu schaffen. Ein kontinuierliches Monitoring ist auch in Zukunft erforderlich, um gesundheitsgefährdende Entwicklungen frühzeitig zu erkennen, und Epidemien von Neusensibilisierungen (primäre Prävention) zu verhindern.

litischen Gesichtspunkten wäre damit auch die Notwendigkeit präventiver Intervention prinzipiell belegt. Das vorrangige Ziel der Prävention der ACD ist die Verhinderung der Sensibilisierung - durch freiwillige Marktrücknahme oder Verbot des Allergens oder durch Absenkung der Konzentration. Es handelt sich also um eine noxenbezogene, generelle Primär-Prävention, oder „Verhältnisprävention“, im Unterschied zur individuell-pädagogisch orientierten „Verhaltensprävention“ [2]. Durch die Arbeit des IVDK sollen, um weitere Sensibilisierungen der Bevölkerung zu verhindern, möglichst frühzeitig diejenigen Stoffe ermittelt werden, die solch einschneidende und kostspielige Maßnahmen mit ihrer quantitativen Bedeutung auch rechtfertigen [3]. 


\section{Fragestellungen}

$\nabla$

1. Welche im Prinzip schon länger bekannten Allergene weisen eine Zunahme in der Sensibilisierungsquote auf oder bestätigen mit gleichbleibend hohen Quoten ihre große Bedeutung? (Überwachung der allgemeinen „Allergensituation“)

2. Welche neu entdeckten Allergene sind in einem definierten Beobachtungszeitraum bedeutsam geworden? (Erfassung von „sentinel health events“, d. h. von Befunden mit Warncharakter)

3. Welche Expositionen (Berufe, Tätigkeiten, private oder berufliche Kontaktstoffe) sind als besonderes Risiko für die Induktion einer Sensibilisierung ermittelt worden? (Überwachung von Expositionen)

\section{Durchfithrung \\ $\nabla$}

Standardisierte Datenerhebung, elektronische Speicherung und zentrale Auswertung: Zunächst werden die Anamnese-Daten (u.a. mit Angaben zum Beruf und zu relevanten Tätigkeiten) und die Ergebnisse des Epikutantests mit einer projekt-eigenen Software erfasst, und dann, nach halbjährlichem Datentransfer, in der Zentrale in Göttingen (Priv.-Doz. Dr. J. Geier) und im IMBE, Erlangen (Prof. Dr. W. Uter), ausgewertet. Bei der Festlegung der einzusetzenden Epikutantests beziehen sich alle IVDK auf die Empfehlungen der DKG [4], mit der seit ihrer Gründung im Mai 1987 durch Prof. Dr. P. J. Frosch, Dortmund, eine enge und fruchtbare Zusammenarbeit besteht. Die Auswertungen folgen den Empfehlungen zur deskriptiven Darstellung und statistischen Analyse multizentrisch erhobener Daten zur ACD [5].

Struktur und Finanzierung der IVDK-Zentrale in Göttingen. Eingebunden in den BMFT-Förderschwerpunkt „Epidemiologie der Allergien“ (im April 1987), wurden Aufbau und Arbeit des IVDK von 11/88 bis 12/93 durch den BMFT gefördert. Für die weitere Finanzierung wurde 1993 eine Fördergesellschaft, die G.E.P.A. e.V. (jetzt IVDK e.V.) gegründet, der Industrieverbände und Einzelunternehmen beitraten. Die gleichzeitige Förderung durch Ministerien, Behörden und Berufsgenossenschaften war und ist eine wesentliche Stütze des IVDK. Der Senat der Georg-AugustUniversität Göttingen hat die Zentrale des IVDK als „außeruniversitäre, mit der Universität kooperierende Einrichtung“" (sog. An-Institut) anerkannt. Örtlich blieb die Einrichtung an die Univ.-Hautklinik angebunden. In dem Institut arbeiten neben einer Sekretärin 5 Akademiker.

\section{Ergebnisse}

Entsprechend der Art der Datengewinnung - halbjährliche Datenlieferung der Partnerkliniken an die Zentrale, und kontinuierlich wachsender Datenbestand, der im August 2008 Daten von 172830 Patienten umfasste - lassen sich zwei Arten der Datennutzung unterscheiden: Nutzung als dynamisches, mit dem Faktor Zeit arbeitendes Überwachungssystem, und Nutzung als ein zu einem gewissen Zeitpunkt bestehendes (oder von einem Ausgangs- zu einem Endpunkt definiertes) statisches Register. Beide Bereiche jedoch dienen vorrangig dem Ziel, das zu erreichen der IVDK sich zur Aufgabe gemacht hat: die Prävention der Kontaktallergie.
Tab. 1 IVDK als Register: Berufsspektrum eines Allergens: Berufe bzw. Berufsgruppen mit einem erhöhten Risiko einer Sensibilisierung (ausgedrückt durch die PR) gegen den Thiuram-Mix, Epoxidharz und Kaliumdichromat nach multipler Poisson Regressionsanalyse [7].

\begin{tabular}{|lll|}
\hline Allergen: Gummi (Thiuram-Mix) & & \\
\hline Beruf & PR & $(95 \% \mathrm{KI})$ \\
\hline Gummihersteller, Vulkaniseur & 4,49 & $(1,6-11,0)$ \\
\hline Arzt, Zahnarzt u. ä. & 2,43 & $(1,5-4,3)$ \\
\hline Krankenpfleger & 2,09 & $(1,3-3,6)$ \\
\hline $\begin{array}{l}\text { Bauarbeiter } \\
\text { Allergen: Epoxidharz }\end{array}$ & 1,90 & $(1,1-3,4)$ \\
\hline $\begin{array}{l}\text { Berufsobergruppe } \\
\text { Bau- und Bergbauberufe }\end{array}$ & PR & $(95 \% \mathrm{KI})$ \\
\hline Maler, Tischler, Keramiker & 4,08 & $(2,8-6,0)$ \\
\hline Chemieberufe & 3,76 & $(2,5-5,6)$ \\
\hline Metallarbeiter & 2,70 & $(1,7-4,2)$ \\
\hline Allergen: Chromat & 1,43 & $(0,99-2,1)$ \\
\hline Beruf & & \\
\hline Bauarbeiter & PR & $95 \% \mathrm{KI}$ \\
\hline Metalloberflächen-Bearbeiter & 3,79 & $(3,2-4,5)$ \\
\hline Metallerzeuger, Former, Gießer etc. & 3,07 & $(1,8-4,8)$ \\
\hline Bergmann, Steinmetz & 2,03 & $(1,2-3,2)$ \\
\hline PR:Praval & 2,02 & $(1,1-3,3)$ \\
\hline
\end{tabular}

PR: Prävalenzratio; KI: Konfidenzintervall.

\section{Der IVDK als Register}

Analysen, die Registerdaten nutzen, können Risikofaktoren identifizieren bzw. Risiken quantifizieren [6]. Zum Beispiel unterliegen bestimmte Berufe dem Risiko (mehr oder weniger) spezifischer Sensibilisierungen ( $\bullet$ Tab. 1). Für den gegen ein bestimmtes Allergen sensibilisierten Arbeitnehmer ist es bei seiner Suche nach alternativen Berufsfeldern von Bedeutung, die Berufe zu kennen, in denen sein Allergen eine große Rolle spielt. Dies ist für eine wirksame Sekundärprävention eine unabdingbare Voraussetzung, und wir haben dazu im Auftrag der Bundesanstalt für Arbeitsschutz und Arbeitsmedizin eine umfangreiche Untersuchung durchgeführt [7]. Als bedeutendster Risikofaktor für eine Sensibilisierung - bedeutender als der Beruf, das Geschlecht, oder eine atopische Dermatitis - wurde von uns die Zahl weiterer Sensibilisierungen (Polysensibilisierung/multiple Sensibilisierung) eindeutig identifiziert [8]. Mit der Kenntnis dieses Risiko-Indikators wäre es möglich, besonders gefährdete Menschen vor (weiteren) Sensibilisierungen zu warnen, im Sinne einer individuellen Primärprävention (d.h. Sensibilisierungsprävention), nämlich diejenigen, die bereits gegen mehrere, vor allem auch schwache, Allergene sensibilisiert sind.

Durch Verknüpfung mit externen Datenquellen, zum Beispiel den Verordnungszahlen von Medikamenten, die uns vom wissenschaftlichen Institut der Ortskrankenkassen (WIdO, Bonn) bereitgestellt wurden, lassen sich Risiken, die mit der Verwendung bestimmter topischer Medikamente verbunden sind, quantifizieren [9]. Beim Vergleich von ophthalmologischen Arzneistoffen mit ähnlichem Indikationsspektrum ist zu erkennen, dass allein auf Basis klinischer Daten Gentamicin als etwas häufigeres Allergen erscheint, unter Berücksichtigung der Exposition, als DDD angegeben, d.h. der pro Tag durchschnittlich verwendeten Wirkstoffmenge, Kanamycin jedoch ein tendenziell höheres Kontaktallergie-Risiko aufweist ( Tab. 2). 


\begin{tabular}{|c|c|c|c|c|c|c|}
\hline Allergen & $\begin{array}{l}\text { Konz } \\
\text { (\%) }\end{array}$ & $\begin{array}{l}\text { IVDK-Daten } \\
\text { \% KA }\end{array}$ & Fälle p.a. & $\begin{array}{l}\text { Verordnungen } \\
\text { (Mio. DDD) p.a. }\end{array}$ & $\begin{array}{l}\text { RI (95\% KI) (Fälle/ } \\
100000 \text { DDD/Jahr) }\end{array}$ & $\begin{array}{l}\text { Tab. } 2 \text { IVDK als Register: } \\
\text { Ausgewählte Epikutantest- }\end{array}$ \\
\hline Pilocarpin- $\mathrm{HCl}$ & 1 & 0,4 & 169 & 59,6 & $0,3(0,0-0,7)$ & ergebnisse des IVDK und Popu- \\
\hline Kanamycinsulfat & 10 & 2,9 & 1336 & 15,4 & $8,7(3,7-13,7)$ & Inzidenz) von ACD gegen Wirk- \\
\hline Gentamicinsulfat* & 20 & 3,5 & 2077 & 43,3 & $4,8(2,5-7,1)$ & stoffe der Ophthalmika-Reihe \\
\hline Chloramphenicol & 5 & 0,9 & 436 & 10,4 & $4,0(0,0-8,7)$ & $(1995-2004)[9]$. \\
\hline
\end{tabular}

Tab. 3 Der IVDK als (aktives*) Überwachungssystem: Strategien der Überwachung und Beispiele.

\begin{tabular}{|c|c|c|}
\hline $\begin{array}{l}\text { Persistenz } \\
\text { eines Problems }\end{array}$ & $\begin{array}{l}\text { Jede halbjährlich erstellte } \\
\text { „Hitliste“ belegt ggf. die } \\
\text { Persistenz von Problemen. }\end{array}$ & (॰ Tab. 4) \\
\hline $\begin{array}{l}\text { Relativierung } \\
\text { eines Problems }\end{array}$ & $\begin{array}{l}\text { Generell in ihrer Bedeutung } \\
\text { überschätzte Allergene, z. B. } \\
\text { Propylenglykol, Isopropyl- } \\
\text { myristat, einige Duftstoffe }\end{array}$ & {$[20-22]$} \\
\hline $\begin{array}{l}\text { Identifizierung } \\
\text { neuer Probleme }\end{array}$ & $\begin{array}{l}\text { Duftstoffe in Kühlschmier- } \\
\text { mitteln } \\
\text { Konservierungsmittel in } \\
\text { Dispersionsfarben } \\
\text { Duftstoff Majantol, }\end{array}$ & $\begin{array}{l}{[23]} \\
{[24]}\end{array}$ \\
\hline $\begin{array}{l}\text { Erkennung von } \\
\text { Trends }\end{array}$ & $\begin{array}{l}\text { Duftstoffe, MDBGN, } \\
\text { Haarfarben }\end{array}$ & $\begin{array}{l}\text { Abb. } 1 \\
{[26-29]}\end{array}$ \\
\hline $\begin{array}{l}\text { Nachweis des } \\
\text { Erfolgs von } \\
\text { Interventionen }\end{array}$ & $\begin{array}{l}\text { Nickel, MDBGN, GMTG, } \\
\text { MCI/MI, Duftstoffe }\end{array}$ & (0 Tab. 5) [2] \\
\hline \multicolumn{3}{|c|}{$\begin{array}{l}\text { MDBGN: Methyldibromoglutaronitril, GMTG: Glycerylmonothioglykolat, } \\
\text { MCI/MI: Methyl(chlor)isothiazolinon. } \\
\text { * Bei einem aktiven Überwachungssystem werden die Falldaten „aktiv“ } \\
\text { eingesammelt (wie beim IVDK), bei passiven Überwachungssystemen wie } \\
\text { z. B. zu (meldepflichtigen) Infektionskrankheiten, Berufskrankheiten oder } \\
\text { Arzneimittelnebenwirkungen wartet das Überwachungssystem („passiv“) } \\
\text { auf den Eingang der Meldungen. Passive Überwachungssysteme leiden } \\
\text { unter einem massiven „Underreporting“, wie im Fall einer Arzneimittel- } \\
\text { nebenwirkung exemplarisch gezeigt werden konnte [30]. }\end{array}$} \\
\hline
\end{tabular}

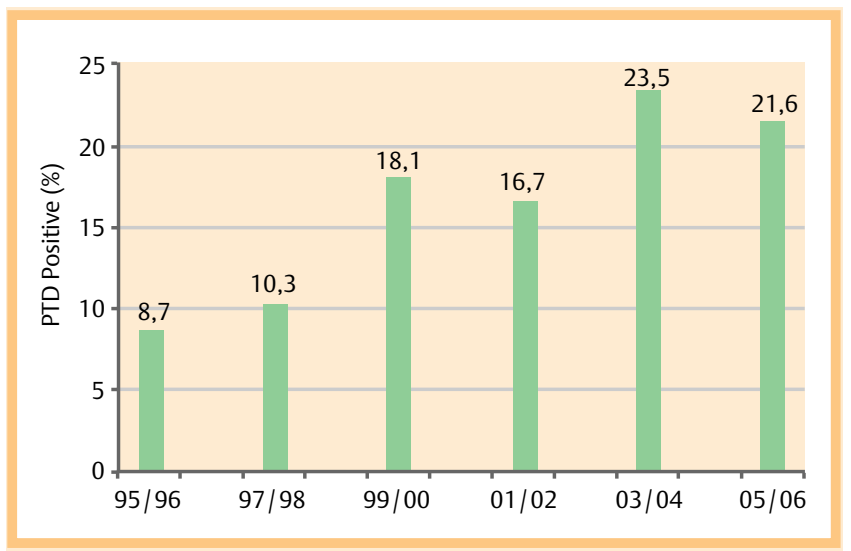

Abb. 1 Sensibilisierung auf die Haarfarbe p-Toluylendiamin (PTD) bei Friseurkundinnen, die epikutan getestet worden waren (IFDK 1995-2006) $[28,29]$. Im Vergleich zu diesem hochselektiven Kollektiv wird die auf das Haarfärben zurückführende Sensibilisierungsprävalenz gegenüber paraPhenylendiamin (mit PTD gekoppelt) in der Allgemeinbevölkerung auf $0,1 \%$ geschätzt [6].
Tab. 4 Allergen Hitliste* des IVDK 2005-2007. Zahl der Getesteten und nach Qualitätsprüfung [12] Berücksichtigten: 2005 ( $\mathrm{n}>$ 9000), 2006 ( $n \sim 10000$ ) und 2007 ( $n>10000$ ). Quoten nach Alter und Geschlecht standardisiert. Reaktionen an Tag 3 oder Tag 4, sofern nicht an Tag 3 abgelesen (Stand der Datenbank: 20.02.2008).

\begin{tabular}{|c|c|c|c|}
\hline Allergen & 2005 & 2006 & 2007 \\
\hline Nickelsulfat & $17,1 \%$ & $17,7 \%$ & $17,3 \%$ \\
\hline Kobaltchlorid & $6,5 \%$ & $7,6 \%$ & $7,5 \%$ \\
\hline Perubalsam & $6,6 \%$ & $7,1 \%$ & $6,8 \%$ \\
\hline Duftstoff-Mix & $6,8 \%$ & $6,6 \%$ & $6,6 \%$ \\
\hline Kaliumdichromat & $5,1 \%$ & $6,2 \%$ & $6,1 \%$ \\
\hline Duftstoff-Mix II & $4,3 \%$ & $4,9 \%$ & $4,6 \%$ \\
\hline $\operatorname{MDBGN}^{* *}(0,3 \%$ Vas, $)$ & $4,9 \%$ & $4,8 \%$ & $3,9 \%$ \\
\hline Männer & $5,3 \%$ & $5,7 \%$ & $4,2 \%$ \\
\hline Frauen & $4,7 \%$ & $4,3 \%$ & $3,8 \%$ \\
\hline Kolophonium & $4,5 \%$ & $4,7 \%$ & $4,0 \%$ \\
\hline Lyral & $2,5 \%$ & $2,6 \%$ & $2,2 \%$ \\
\hline Propolis & $2,6 \%$ & $2,4 \%$ & $2,2 \%$ \\
\hline Terpentin & $1,3 \%$ & $2,4 \%$ & $1,8 \%$ \\
\hline MCI/MI (Kathon CG) & $2,1 \%$ & $2,3 \%$ & $2,1 \%$ \\
\hline Wollwachsalkohole & $2,2 \%$ & $2,1 \%$ & $2,0 \%$ \\
\hline Kompositen-Mix & $2,3 \%$ & $1,9 \%$ & $1,2 \%$ \\
\hline Thiuram-Mix & $2,3 \%$ & $1,9 \%$ & $2,0 \%$ \\
\hline Formaldehyd & $1,5 \%$ & $1,6 \%$ & $1,2 \%$ \\
\hline Epoxidharz & $1,5 \%$ & $1,4 \%$ & $1,4 \%$ \\
\hline Paraben-Mix & $1,5 \%$ & $1,4 \%$ & $1,1 \%$ \\
\hline Bronopol & $1,4 \%$ & $1,3 \%$ & $1,2 \%$ \\
\hline Bufexamac & $1,5 \%$ & $1,3 \%$ & $\S$ \\
\hline \multicolumn{4}{|c|}{$\begin{array}{l}\text { *Wegen selektiver Testung bzw. problematischer Testzubereitung [11] } \\
\text { nicht berücksichtigt: Thiomersal, Amerchol L101, Polyvidon-lod, Cocami- } \\
\text { dopropylbetain, Octylgallat, Quecksilberamid-chlorid, para-Toluylendia- } \\
\text { min, Palladiumchlorid, Propylenglycol, tert Butylhydrochinon. } \\
{ }^{* *} \text { In den Jahren } 2003 \text { und } 2004 \text { wurde Euxyl K } 4001 \% \text { (MDBGN ( } 0,2 \% \text { ) ge- } \\
\text { testet. Die Reaktionsquoten betrugen } 3,2 \text { bzw. } 3,4 \% \text {. Sie waren bei Männern } \\
\text { und Frauen gleich. Möglicherweise geht die (meist) Kosmetika-assoziierte } \\
\text { Sensibilisierung (bei Frauen) zurück. Die Testung von MDBGN 0,2\% im Mo- } \\
\text { nitor-block im Jahre } 2005 \text { ergab lediglich eine Quote von 2,6\%. Die Testung } \\
\text { mit 0,3\% ergibt häufiger „, falsch positive“ Ergebnisse. } \\
\S 2007 \text { nicht in Standardreihe getestet. }\end{array}$} \\
\hline
\end{tabular}

\section{Der IVDK als Überwachungssystem}

Der IVDK versteht sich jedoch vornehmlich als Überwachungssystem, denn dessen Ergebnisse können, anders als im Falle des klinischen Registers, frühzeitig zu präventiven Interventionen führen [3]. Die Strategien der Überwachung betreffen nicht nur die Erkennung ansteigender Trends, obwohl diese sicher die bedeutsamste ist, wird doch dadurch ein "sentinel health event“ (das Warnsignal in einem Überwachungssystem) erkannt ( Tab.3 u. $\bigcirc$ Abb. 1). Auch der Nachweis der Persistenz eines Problems ( $\odot$ Tab. 4) und des Erfolgs von Interventionen ist nicht zu unterschätzen, denn letztere sind aufwendig und kostenintensiv. Der Nachweis rechtfertigt also auch in Zukunft den teilweise erheblichen Aufwand [2]. 
Tab. 5 IVDK als Überwachungssystem: Nachweis des Erfolgs noxenbezogener Interventionen im Bereich der Kontaktallergie durch IVDK-Daten [2].

\begin{tabular}{|c|c|c|c|c|}
\hline Allergen/Umfeld & a & Intervention & b & Ref. \\
\hline $\begin{array}{l}\text { Glycerylmonothio- } \\
\text { glykolat (saure Dauer- } \\
\text { welle) bei jungen } \\
\text { Friseurinnen }\end{array}$ & $46 \%$ & Marktrücknahme & $0 \%$ & {$[31)$} \\
\hline $\begin{array}{l}\text { Nickel } \\
\text { (Modeschmuck) } \\
\text { bei Frauen unter } \\
30 \text { Jahren }\end{array}$ & $37 \%$ & $\begin{array}{l}\text { Nickeldirektive } \\
\text { der EU; Limit } \\
0,5 \mu \mathrm{g} \text { pro } \mathrm{cm}^{2} \\
\text { pro Woche }\end{array}$ & $26 \%$ & [32] \\
\hline $\begin{array}{l}\mathrm{MCI} / \mathrm{MI} \text { bei männ- } \\
\text { lichen Patienten mit } \\
\text { Farben-Exposition }\end{array}$ & $11 \%$ & $\begin{array}{l}\text { Begrenzung auf } \\
15 \text { ppm durch EU } \\
\text { und UBA }\end{array}$ & $4 \%$ & [24] \\
\hline $\begin{array}{l}\text { Duftstoffe } \\
\text { (z. B. Isoeugenol) }\end{array}$ & $13 \%$ & $\begin{array}{l}\text { Reduktion der } \\
\text { Einsatzkonzen- } \\
\text { tration }\end{array}$ & $7 \%$ & [26] \\
\hline
\end{tabular}

\section{Zusammenfassung und Perspektiven \\ $\nabla$}

Neben der hier nicht weiter dargestellten Bearbeitung von Themen der Qualitätssicherung [10-12], der Mitwirkung in europäischen Projekten [13] und der experimentellen Forschung [14 - 16] ist das vorrangige Ziel des IVDK die Prävention von Kontakt-Allergien. Die durch den IVDK bereitgestellten Informationen unterstützen sowohl die Hersteller von Gebrauchsgütern [17] und den betrieblichen Arbeitsschutz als auch die regulatorischen Behörden in ihren Bemühungen um sichere Produkte und Arbeitsstoffe [18,19]:

a) Durch Erkennung (IVDK) und Beseitigung (Verbot oder freiwillige Beschränkung) einer Noxe werden schon die Sensibilisierungen verhindert (generelle Primärprävention als „Königin der Prävention“).

b) Durch Erkennung der wichtigen Allergene (IVDK) und Deklaration (regulatorische Entscheidung) können bereits sensibilisierte Verbraucher und Arbeitnehmer informiert und geschützt werden (Sekundärprävention und Rehabilitation).

Die Bevölkerung bleibt auch in Zukunft allergenen Stoffen ausgesetzt. Ein kontinuierliches Monitoring ist erforderlich, um gesundheitsgefährdende Entwicklungen frühzeitig zu erkennen. Dies ist umso dringlicher, als in naher Zukunft prädiktive Tierversuche zur Erfassung sensibilisierender Eigenschaften neuer Stoffe verboten sein werden und somit ein „Humanexperiment“ großen Stils droht.

\section{Abstract}

\section{The IVDK - A Short Synopsis}

$\nabla$

Fifty dermatology departments from Germany, Austria and Switzerland collaborate to study the clinical epidemiology of contact allergies (CA). Data generated in the course of the diagnostic work-up of CA (e.g. patch test data) are stored since 1989 in the data centre in Göttingen, amounting to more than 170000 patients (August 2008). These data can be used as a register, and as a surveillance system. Analysis of the register may identify and quantify risk factors of sensitization to an allergen, which is exemplified with the case of the aminoglycoside antibiotics. It turned out that kanamycin is associated with a higher risk of sensitization than gentamicin. In contrast, data collected every 6 months (from $\sim 6000$ patients) allow for time trend analyses of allergens, thus identifying allergens of concern, which is of utmost importance for early preventive intervention. Here, the epidemiology of allergies to fragrances and other allergens serve as examples. Continuous monitoring of contact allergens will also be mandatory in future, as the CA pre-marketing screening systems will have imperfect predictive values with regard to human CA risk.

\section{Literatur}

1 Schnuch A, Uter W. Die Verbreitung des Kontaktekzems in der Allgemeinbevölkerung und in verschiedenen Berufen. In: Schultze-Werninghaus G, Fuchs T, Bachert C, Wahn U (Hrsg). Manuale allergologicum. München: Dustri, 2004: 297-345

2 Uter W, Geier J, Schnuch A. Der Erfolg noxenbezogener Primärprävention. Nachweis am Beispiel der Kontaktallergie-Überwachung. Gefahrstoffe - Reinhaltung der Luft 2008; 68: 202 - 208

3 Schnuch A, Uter W, Geier J et al. Überwachung der Kontaktallergie: Zur „Wächterfunktion“ des IVDK. Allergo J 2005; 14: 618-629

4 Schnuch A, Aberer W, Agathos M et al. Durchführung des Epikutantests mit Kontaktallergenen. Leitlinien der Deutschen Dermatologischen Gesellschaft (DDG) und der Deutschen Gesellschaft für Allergie und klinische Immunologie (DGAKI). JDDG 2008; 6: 770-775

5 Uter W, Schnuch A, Gefeller O. Guidelines for the descriptive presentation and statistical analysis of contact allergy data. Contact Dermatitis 2004; 51: 47-56

6 Schnuch A, Lessmann H, Frosch PJ, Uter W. Para-Phenylenediamine: the profile of an important allergen. Results of the IVDK. Br J Dermatol 2008; 159: 379-386

7 Uter W, Gefeller O, Geier J et al. Untersuchungen zur Abhängigkeit der Sensibilisierung gegen wichtige Allergene von arbeitsbedingten sowie individuellen Faktoren. Schriftenreihe der Bundesanstalt für Arbeitsschutz und Arbeitsmedizin Wirtschaftsverlag NW, Bremerhaven 2002; Fb 949

8 Schnuch A, Brasch J, Uter W. Polysensitization and increased susceptibility in contact allergy: a review. Allergy 2008; 63: 156-167

9 Menezes de Padua C, Nink K, Schnuch A et al. Kontaktallergie gegen topische Arzneistoffe - epidemiologische Risikoabschätzung. Allergo J 2008; 17: $88-89$

10 Geier J, Uter W, Schnuch A, Brasch J. Diagnostic screening for contact allergy to mercaptobenzothiazole derivatives. Am J Contact Dermatitis 2002; $13: 66-70$

11 Geier J, Uter W, Lessmann H, Schnuch A. The positivity ratio - another parameter to assess the diagnostic quality of a patch test preparation. Contact Dermatitis 2003; 48: 280-282

12 Uter W, Mackiewicz M, Schnuch A, Geier J. Interne Qualitätssicherung von Epikutantest-Daten des multizentrischen Projektes „Informationsverbund Dermatologischer Kliniken“ (IVDK). Dermatologie in Beruf und Umwelt 2005; 53: 107 - 114

13 Hegewald J, Schnuch A, Uter W. Klinische Kontaktallergie-Surveillance in Europa - das ESSCA-Netzwerk. Allergo J 2008; 17: 86-87

14 Schnuch A, Westphal GA, Müller MM et al. Genotype and phenotype of $\mathrm{N}$-acetyltransferase 2 (NAT2) polymorphism in patients with contact allergy. Contact Dermatitis 1998; 38: 209-211

15 Reich K, Westphal G, König IR et al. Association of allergic contact dermatitis with a promoter polymorphism in the IL16 gene. J All Clin Immunol 2003; 112: 1191 - 1194

16 Westphal G-A, Schnuch A, Schulz T-G et al. Homozygous gene deletions of the glutathione S-transferases M1 and T1 are associated with thimerosal sensitization. Int Arch Occup Environ Health 2000; 73: $384-$ 388

17 Geier J, Lessmann H, Schnuch A, Fuchs Th. Kontaktallergien durch Formaldehyd abspaltende Biozide. Allergologie 1997; 20: 215- 224

18 Schnuch A, Geier J, Lessmann H, Uter W. Untersuchungen zur Verbreitung umweltbedingter Kontaktallergien mit Schwerpunkt im privaten Bereich. Im Auftrag des Umweltbundesamtes (FKZ 29961 219). WaBoLu 2004; Nr. 01; 04 (ISSN 0175-4211)

19 Geier J, Uter W, Lessmann H, Schnuch A. „Frühzeitige Erkennung allergener Stoffe bei beruflicher und nicht-beruflicher Exposition“ (FaSt). Forschungsvorhaben des HVBG, der BGW und der BG-Bau. Abschluß- 
bericht des IVDK. Sankt Augustin: Hauptverband der Gewerblichen Berufsgenossenschaften, 2002

20 Lessmann H, Schnuch A, Geier J, Uter W. Skin-sensitizing and irritant properties of propylene glycol. Contact Dermatitis 2005; 53: 247-259

21 Uter W, Schnuch A, Geier J, Lessmann H. Isopropyl myristate recommended for aimed rather than routine patch testing. Contact Dermatitis 2004; 50: $242-244$

22 Schnuch A, Geier J, Uter W, Frosch PJ. Sensitization to 26 fragrances to be labelled according to current European regulation. Contact Dermatitis 2007; 57: 1 - 10

23 Geier J, Lessmann H, Schnuch A, Uter W. Contact sensitization in metalworkers with occupational dermatitis exposed to water-based metalworking fluids. Results of the research project „FaSt“. Int Arch Occup Environ Health 2004; 77: $543-551$

24 Schnuch A, Uter W, Geier J et al. Kontaktallergien gegen Dispersionsfarben. Epidemiologische Überwachung durch den IVDK, Intervention des Umweltbundesamtes und erfolgreiche Primärprävention. Allergo J 2002; 11: 39-47

25 Schnuch A, Geier J, Uter W, Frosch PJ. Majantol - a new important fragrance allergen. Contact Dermatitis 2007; 57: 48 - 50

26 Schnuch A, Lessmann H, Geier J et al. Contact allergy to fragrances: frequencies of sensitization from 1996 to 2002. Results of the IVDK. Contact Dermatitis 2004; 50: 65-76
27 Geier J, Schnuch A, Fuchs T. Zunahme der Kontaktallergien gegen Methyldibromoglutaronitril in Deutschland. Allergologie 1996; 19: 399402

28 Uter W, Lessmann H, Geier J, Schnuch A. Contact allergy to ingredients of hair cosmetics in female hairdressers and clients - an 8-year analysis of IVDK data. Contact Dermatitis 2003; 49: 236-240

29 Uter W, Lessmann H, Geier J, Schnuch A. Aktuelle Sensibilisierungshäufigkeiten gegen Haarkosmetik-Kontaktallergene bei Friseuren und Kundinnen - Ergebnisse des IVDK 2003 -2006. JDDG 2007; 5: 993 1001

30 Schnuch A, Geier J, Gefeller O, Uter W. Eine heimtückische und häufige Nebenwirkung: Das Ekzemtherapeuticum Bufexamac verursacht selbst Kontaktallergien. Ergebnisse des IVDK. Dtsch Med Wochenschr 2005; 130: 2881 - 2886

31 Uter W, Geier J, Lessmann H, Schnuch A. Is contact allergy to glyceryl monothioglycolate still a problem in Germany? Contact Dermatitis 2006; 55: 54-56

32 Schnuch A, Geier J, Lessmann H, Uter W. Rückgang der Nickelkontaktallergie in den letzten Jahren. Eine Folge der „Nickel-Verordnung“? Auswertungen der Daten des IVDK der Jahre 1992-2001. Hautarzt 2003; 54: $626-632$ 